\title{
PHOTOMETRY OF ECLIPSING BINARY STARS IN THE LARGE AND SMALL MAGELLANIC CLOUDS
}

\author{
G. P. Bayne,${ }^{1}$ W. Tobin,${ }^{1}$ J. D. Pritchard,${ }^{2}$ I. Bond,${ }^{3}$ K. R. Pollard, ${ }^{1}$ S. C. Besier, ${ }^{1}$ S. Noda,${ }^{4}$ T. Sumi, ${ }^{4}$ T. \\ Yanagisawa, ${ }^{4}$ M. Sekiguchi, ${ }^{5}$ M. Honda,${ }^{5}$ Y. Muraki, ${ }^{4}$ M. Takeuti, ${ }^{6}$ J. B. Hearnshaw,${ }^{1}$ P. M. Kilmartin, ${ }^{1}$ R. J. \\ Dodd, ${ }^{7}$ D. J. Sullivan, ${ }^{8}$ and P. C. M. Yock ${ }^{9}$
}

\section{RESUMEN}

Se han obtenido curvas de luz para tres binarias eclipsantes en las Nubes de Magallanes mediante fotometría CCD $u V_{\mathrm{J}} I_{\mathrm{C}}$. Uno de los objetos en la GNM, MACHO*05:36:48.7-69:17:00, es un sistema excéntrico, con $e$ $=0.20$ y un período de $3.853534 \pm 0.000005 \mathrm{~d}$. Las soluciones iniciales indican una primaria con temperatura en el intervalo $T_{\text {eff, } 1}=20,000-35,000 \mathrm{~K}, \mathrm{y}$ una secundaria con una $T_{\text {eff }, 2} 1000-2000 \mathrm{~K}$ más fría que la primaria, con inclinaciones entre $i=84.2^{\circ}-86.0^{\circ}$. Dos objetos en la PNM, MOA J005018.4-723855 and MOA J005623.5-722123, tienen períodos de $1.8399 \pm 0.0004$ y $2.3199 \pm 0.0003$ días, respectivamente. Ambos tienen órbitas circulares, y el primero es un sistema semi-separado.

\section{ABSTRACT}

The light curves for three eclipsing binaries in the Magellanic Clouds have been obtained using CCD $u V_{\mathrm{J}} I_{\mathrm{C}}$ photometry. One target in the LMC, MACHO*05:36:48.7-69:17:00, is an eccentric system, $e=0.20$, with a period of $3.853534 \pm 0.000005 \mathrm{~d}$. Initial solutions indicate a primary component in the range $T_{\text {eff, } 1}=20,000-35,000 \mathrm{~K}$ and the secondary $T_{\text {eff }, 2} 1000-2000 \mathrm{~K}$ cooler than the primary, with inclinations ranging $i=84.2^{\circ}-86.0^{\circ}$. Two targets in the SMC, MOA J005018.4-723855 and MOA J005623.5-722123, have periods of $1.8399 \pm 0.0004$ and $2.3199 \pm 0.0003$ days respectively. Both have circular orbits with the former being a semi-detached system.

\section{Key Words: BINARIES: ECLIPSING — STARS: EARLY-TYPE — STARS: FUNDAMENTAL PARAM- ETERS - STARS: INDIVIDUAL (MACHO*05:36:48.7-69:17:00, MOA J005018.4-723855, MOA J005623.5-722123)}

\section{GENERAL}

Studies of extra-galactic eclipsing binary stars are currently of interest for two reasons. First, if the observational material is of sufficient quality, they permit high-accuracy testing of models of stellar evolution calculated for metallicities other than those found in our own Galaxy, and essentially let us understand single stars. Second, they yield direct distances, which hold the promise of being important calibrators for the extra-galactic distance scale, though observational and analytical uncertainties still need to be reduced.

\footnotetext{
${ }^{1}$ Department of Physics and Astronomy, University of Canterbury, Christchurch, New Zealand.

${ }^{2}$ European Southern Observatory, Santiago, Chile.

${ }^{3}$ Royal Observatory, Edinburgh, UK.

${ }^{4}$ Solar Terrestrial Environment Laboratory, Nagoya University, Nagoya.

${ }^{5}$ Institute of Cosmic Ray Research, University of Tokyo, Tanashi, Tokyo, Japan.

${ }^{6}$ Tohoku University Astronomical Institute, Sendai, Japan

${ }^{7}$ Carter National Observatory, Wellington, New Zealand.

${ }^{8}$ School of Chemical and Physical Sciences, Victoria University of Wellington, Wellington, New Zealand.

${ }^{9}$ Science Faculty, University of Auckland, Auckland, New Zealand.
}

One method of distance determination is via the relation in equations 1 and 2 .

$$
\begin{aligned}
m_{\mathrm{V}}-M_{\mathrm{V}} & =m_{\mathrm{V}}-A_{\mathrm{V}}-M_{\text {bol }_{\odot}}+ \\
& +5 \log \left(\frac{R}{R_{\odot}}\right)+10 \log \left(\frac{T_{\mathrm{eff}}}{T_{\mathrm{ef}_{\odot}}}\right)+B C
\end{aligned}
$$

and

$$
\left(\frac{R}{R_{\odot}}\right)=\frac{r 1.9577 \times 10^{-2}\left(K_{1}+K_{2}\right) P(1-e)^{\frac{1}{2}}}{\sin i}
$$

The parameters derived from photometric observations and analyses are period, $P$, eccentricity $e$, relative radius $r$, extinction $A_{\mathrm{V}}$ and orbital inclination $i$. The spectroscopic observations provide the orbital semi-major axis $a$, which scales the system, radial velocity semi-amplitudes $K_{1}$ and $K_{2}$, and the mass ratio, $q$. Spectrophotometry is also required to constrain effective temperatures and reddening. All parameters are required in order to derive a com- 
plete solution for the eclipsing binary system. Here we present the time-series photometric data obtained at the Mount John University Observatory, MJUO, the reduction and initial analysis. The goal is to obtain photometric accuracy of least $1 \%$ and sufficient phase coverage for accurate light curve fitting and analysis.

\section{TARGET SELECTION}

Detecting eclipsing binaries is an essential prerequisite to selecting interesting systems for detailed study. A number of large times-series photometric databases have been collated by groups such as EROS, MACHO, MOA and OGLE, thereby providing observations for millions of stars over many years. Data-mining of these databases has yielded catalogues of many variable types, thereby enabling a more stringent selection of possible candidates for follow-up detailed study.

The LMC target, MACHO*05:36:48.7-69:17:00 was selected from the MACHO catalogue of eclipsing binaries in the LMC (Alcock et al. 1997). The system did not appear to suffer from any significant blending with nearby stars. The relative brightness of the system, together with its deep, well detached eclipses, made it an ideal candidate, especially for comparison to theoretical models. It is located near the outer regions of the Tarantula nebula, close to SN1987A.

The MOA (Microlensing Observations in Astrophysics) group, based at MJUO, provided their SMC database to search through for eclipsing binaries. The search resulted in the catalogue of 169 eclipsing binaries in the SMC (Bayne et al. 2002), from which two targets were selected. The fields observed by the MOA project included outer less dense stellar regions of the SMC, and therefore gave the possibility of selecting relatively bright systems where blending is not so severe.

The MJUO site enables observations of the Magellanic Clouds all year round, with various airmasses, typically ranging from 1.1 to 2.3 . Therefore targets in both the LMC and SMC are desirable to optimize the quality and efficiency of the data collection.

\section{OBSERVATIONS}

The photometric observations were made between April 1999 and July 2002 using the $1 \mathrm{~m}$ telescope at MJUO in $f / 7.8$. Observations were made using a SITe $003 \mathrm{AB} \mathrm{CCD}, 1024 \times 1024$ pixel array, $24 \mu \mathrm{m}$ pixel size, in conjunction with the CCD photometer head. The observations were in three bandpasses; Strömgren $u$, Johnson $V_{\mathrm{J}}$ and Cousins $I_{\mathrm{C}}$.

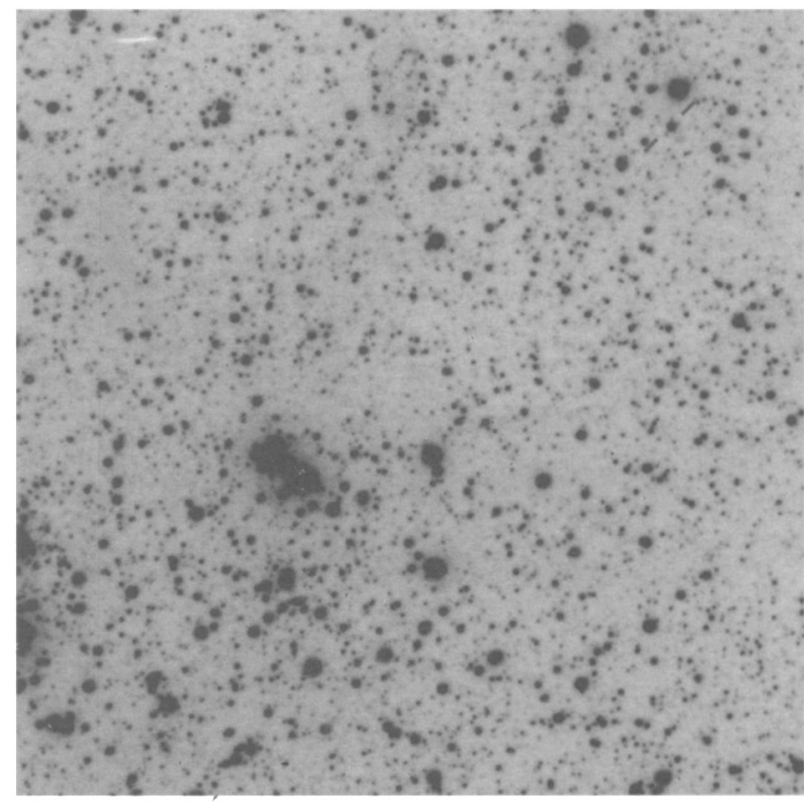

Fig. 1. The $I_{\mathrm{C}}$-bandpass reference frame for MACHO* $^{*} 5: 36: 48.7-69: 17: 00$, with the target indicated in the top right. It was constructed using three stacked images to improve the signal-to-noise ratio.

Typical exposure times were 1200,700 and 400 s respectively. The observations spanned 1268,618 and 682 days for MACHO*05:36:48.7-69:17:00, MOA

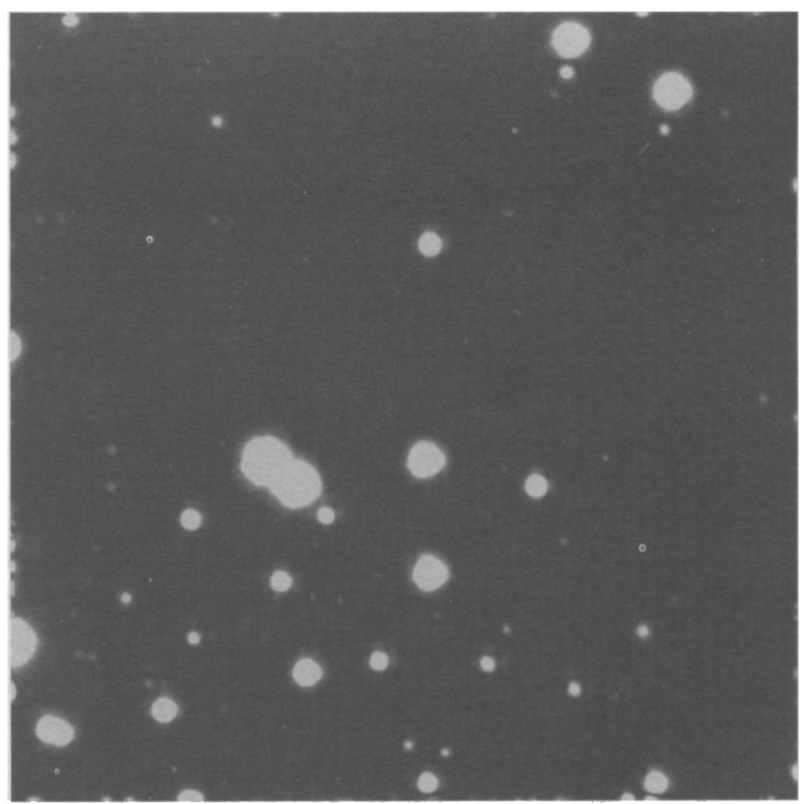

Fig. 2. The frame containing the variables stars in the reference frame of $\mathrm{MACHO}^{*} 05: 36: 48.7-69: 17: 00, I_{\mathrm{C}}$ bandpass with the target indicated in the top right. The large star-like objects are stars that have saturated on the reference frame. 
a

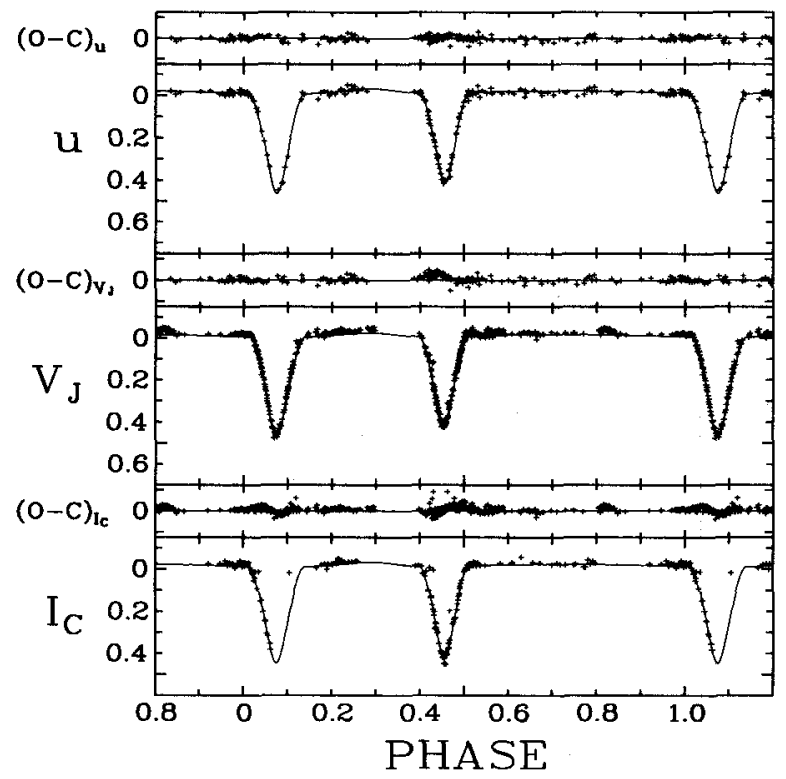

$\mathbf{b}$

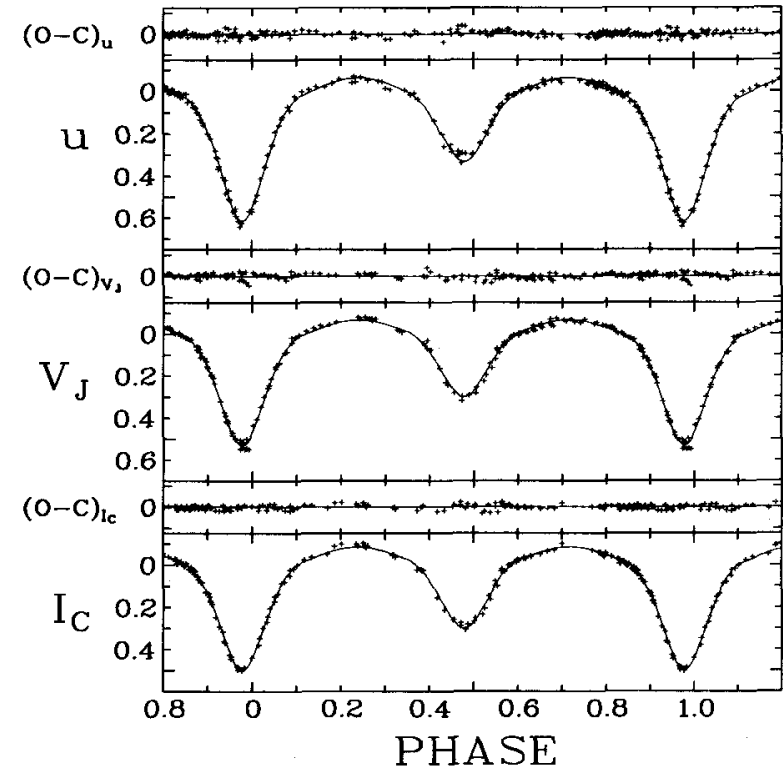

Fig. 3. Light curves for MACHO*05:36:48.7-69:17:00, a, and MOA J005018.4-723855, $\mathbf{b}$ in the three bandpasses $u, V_{\mathbf{J}}$ and $I_{\mathrm{C}}$. The $W D$ fits, solid lines, are overlaid on the phased light curves, + . Immediately above each light curve are the deviations from the fit (Observed-Calculated). These are indicative of a typical solution for each system.

J005018.4-723855 and MOA J005623.5-722123. Initially, more observations were made in the Johnson $V_{\mathrm{J}}$ bandpass for MACHO*05:36:48.7-69:17:00. This gave a different mean epoch for the $V_{\mathrm{J}}$ and $u / I_{\mathrm{C}}$ observations. Improvements in the image quality (symmetry of the stars on the image) were made with the replacement of the old manual guide system with a ST4 auto-guide system.

\section{DATA REDUCTIONS AND ANALYSIS}

An image subtract technique ISIS-2.1 (Alard 2000a, Alard \& Lupton 2001) was employed for the data reduction process, figures 1 and 2, rather than the traditional profile, PSF, fitting techniques such as DAOPHOT II. It has been shown (Alard 2000b) that image subtraction techniques can provide more consistent photometry, especially in crowded fields, as was the case of MJUO observations of EROS 1044. The light curve analysis was performed using flux differences, or a.c. signal as termed by Woźniak (2000). The difficulty in working with a d.c. signal, such as magnitudes, is that the background level is very difficult, if not impossible at times, to determine accurately in crowded fields. In order to obtain an estimate of the d.c. signal, DAOPHOT II was applied to the reference frame. The position and magnitude of the red clump were estimated from the reference frames. They were compared with known values of the red clump in similar regions in both the LMC and SMC. Thus approximate values for the target stars were determined.

A modified version of the Wilson-Devinney code (Wilson 1994), hereafter $W \mathrm{D}$, was used, incorporating an atmosphere routine using Kurucz model atmospheres. The adopted abundances for the $\mathrm{LMC}$ and SMC were $[\mathrm{m} / \mathrm{H}]=-0.3 \&$ -0.5 respectively. Figure 3 presents the light curves of MACHO*05:36:48.7-69:17:00 and MOA J005018.4-723855 respectively in the $u, V_{\mathrm{J}}$ and $I_{\mathrm{C}}$ bandpasses. The $W D$ fits are overlaid with the deviations from the fit (Observations-Calculated), presented immediately above the light curve. The $W D$ analysis assists in providing bounds for the systems based on astrophysically plausible solutions. The light curve analysis for MOA J005623.5-722123 is still in progress.

Light-curve solutions for ranges of mass ratio and primary effective temperature are computed for fixed values of the longitude of periastron. The inclination, $T_{\text {eff,2 }}$ and potentials of the primary and secondary are left to vary. The apparent magnitudes of the systems indicate early B stars; therefore gravity darkening is allowed for in the WD light curve analysis. The results can then be compared to the evolutionary models for these types of stars. In this analysis, we assume distance moduli to the LMC and SMC of 18.50 and $18.90 \mathrm{mag}$ respectively.

For $\mathrm{MACHO}^{*} 05: 36: 48.7-69: 17: 00$, light-curve 
analysis yields a period of $3.853534 \pm 0.000005 \mathrm{~d}$ with $T_{0}=\mathrm{JD} 2449073.381 \pm 0.002$. A possible $W \mathrm{D}$ solution indicates primary $T_{\text {eff }, 1}=27,500 \mathrm{~K}$ and secondary $T_{\text {eff }, 1}=25,000 \mathrm{~K}$, inclination $i=84.4^{\circ}$ and eccentricity $e=0.20$.

MOA J005018.4-723855 has a period of $1.8399 \pm 0.0004 \mathrm{~d}$ and $T_{0}=\mathrm{JD} 2450681.083 \pm 0.003$. The WD analysis indicates a semi-detached system with the secondary filling its Roche lobe. The orbit is circular, $e=0$. The solution oscillates between a well fitted $I_{\mathrm{C}}$ bandpass but poorly fitted $u$ and vice versa. The addition of a third light parameter may be required to obtain a better fit. This target is the most affected by blending.

A period of $2.3199 \pm 0.0003 \mathrm{~d}$, with $T_{0}=$ JD 2450681.934 \pm 0.004 was determined for MOA J005623.5-722123. The light curve indicates a circular orbit, $e=0$. The $W \mathrm{D}$ analysis is not yet completed for this target.

\section{CONCLUSIONS}

We were able to obtain approximately $2 \%$ photometric accuracy, compared to our initial goal of at least $1 \%$. Further observations in better conditions may improve the level of photometric accuracy; however, the effects of blending, especially in the two MOA targets, have yet to be fully explored. Calibration in a standard photometric system is required for these targets. MACHO*05:36:48.7-69:17:00 shows promise as a distance indicator, provided that spectroscopy and spectrophotometry can be acquired.

GB acknowledges the financial support of the Dennis William Moore Fund, and an IAU travel grant for attendance of the IAU Colloquium 191.

\section{REFERENCES}

Alard, C. 2000a, A\&AS, 144, 363

Alard, C. 2000b, in ASP Conf. Ser. 203, The Impact of Large-Scale Surveys on Pulsating Star Research, ed. L. Szabados L.\& D. Kurtz (IAU Coll. 176) (San Francisco: ASP), 50

Alard, C., \& Lupton, R. H. 2001, ApJ, 503, 325

Alcock, C. et al. 1997, AJ, 114, 326

Bayne, G. P. et al. 2002, MNRAS, 331, 609

Wilson, R. E. 1994, PASP, 106, 921

Woźniak, P. R. 2000, Acta Astronomica, 50, 421

Glenn P. Bayne, S. C. Besier, J. B. Hearnshaw, P. M. Kilmartin, K. R. Pollard, and W. Tobin: Department of Physics and Astronomy, University of Canterbury, Private Bag 4800, Christchurch, New Zealand (g.bayne, s.besier,j.hearnshaw,p.kilmartin,k.pollard,w.tobin@phys.canterbury.ac.nz).

J. D. Pritchard: European Southern Observatory, Casilla 19001, Santiago, Chile (jpritchard@eso.org).

I. Bond: Royal Observatory, Edinburgh, Blackford Hill, Edinburgh, EH9 3HJ, UK (iab@roe.ac.uk).

Y. Muraki, S. Noda, T. Sumi, T. Yanagisawa: Solar Terrestrial Environment Laboratory, Nagoya University, Nagoya 464, Japan.

M. Honda, M. Sekiguchi: Institute of Cosmic Ray Research, University of Tokyo, Tanashi, Tokyo 188, Japan.

M. Takeuti: Tohoku University Astronomical Institute, Sendai 980, Japan.

R. J. Dodd: Carter National Observatory, P.O. Box 2909, Wellington, New Zealand (Richard.Dodd@vuw.ac.nz).

D. J. Sullivan: School of Chemical and Physical Sciences, Victoria University of Wellington, P.O. Box 600, Wellington, New Zealand (denis.sullivan@vuw.ac.nz).

P. C. M. Yock: Science Faculty, University of Auckland, Private Bag 92019, Auckland, New Zealand (p.yock@ auckland.ac.nz). 


\section{DISCUSSION}

Kaper - How accurately will the distance to the LMC and the SMC be determined, e.g., in comparison to the Cepheid distance method?

Bayne - With very careful analysis, we believe we can obtain distances to within a few percent to the LMC. The reddening can be determined for each object, this is a main source of error in other methods. For the Cepheid distance, different analysis methods are giving different results. Eclipsing binaries can be used for depth determination.

Scarfe - Can you place your systems on a colour-magnitude diagram for us? What are their absolute magnitudes?

Bayne - For LMC target MACHO 053648.7 - 691659.95 about 15.25 mag. For SMC target MOA 00501840 - 7238552 about $15.8 \mathrm{mag}$ (but I would have to look up the values).

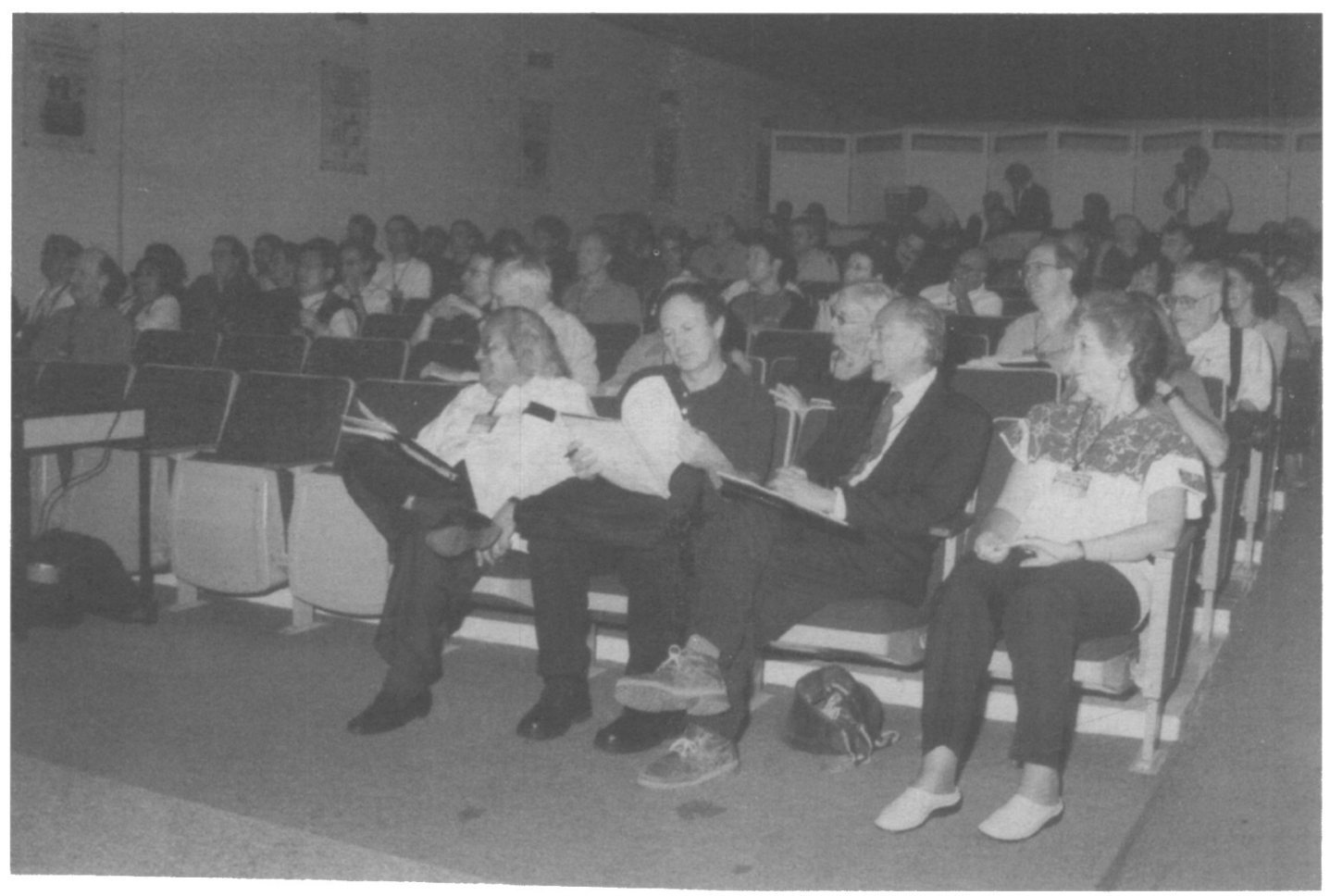

Arcadio Poveda, Ed Guinan, Kam-Ching Leung and Christine Allen. 